\title{
Fast Wind-Induced Migration of Leddies in the South China Sea
}

\author{
DORON NOF \\ Department of Earth, Ocean and Atmospheric Sciences, and Geophysical Fluid Dynamics Institute, \\ The Florida State University, Tallahassee, Florida \\ YINGLAI JIA \\ Physical Oceanography Laboratory, Ocean University of China, Qingdao, China, and Center for Ocean \\ and Atmospheric Prediction, The Florida State University, Tallahassee, Florida \\ ERIC CHASSIGNET \\ Department of Earth, Ocean and Atmospheric Sciences, and Center for Ocean and Atmospheric Prediction, \\ The Florida State University, Tallahassee, Florida \\ AlEXANDRA BOZEC \\ Center for Ocean and Atmospheric Prediction, The Florida State University, Tallahassee, Florida
}

(Manuscript received 6 July 2010, in final form 30 March 2011)

\begin{abstract}
Eddies off the Strait of Luzon (termed here as "Leddies," analogous to "Teddies" originating from the Indonesian Throughflow) are formed rapidly and migrate swiftly. Their migration rate $\left(\sim 10-20 \mathrm{~cm} \mathrm{~s}^{-1}\right)$ is an order of magnitude faster than that of most eddies of the same scale $\left(\sim 1 \mathrm{~cm} \mathrm{~s}^{-1}\right)$. On the basis of observations, it has been suggested earlier that the rapid generation process is due to the southeast monsoon.

Here, the authors place this earlier suggestion on a more solid ground by developing both analytical and process-oriented numerical models. Because the eddies are formed by the injection of foreign, lighter Kuroshio water into the South China Sea (SCS), the eddies are modeled as lenses: that is, "bullets" that completely encapsulate the mass anomaly associated with them. It turns out that the rings migrate at an angle $\alpha$ (between $0^{\circ}$ and $90^{\circ}$ ) to the right of the wind direction \{i.e., $\tan ^{-1}\left[(2-\gamma) f^{2} R / 8 g^{\prime} C_{D}\right]$, where in conventional notation $\gamma$ is the vorticity, $R$ the eddy radius, and $C_{D}$ the interfacial friction coefficient along the lower interface of the lens $\}$. Their fast migration speed is given by $2\left(\tau_{S} / \rho_{W}\right)(\sin \alpha) / f H$, where $\tau_{S}$ is the wind stress on the surface, $\rho_{w}$ the water density, and $H$ is the maximum eddy depth. With high interfacial drag (i.e., large $C_{D}$ ), the rings move relatively slowly (but still a lot faster than Rossby waves) in the wind direction, whereas with low drag they move fast at $90^{\circ}$ to the right. These analytically predicted values are in good agreement with isopycnic numerical simulations.
\end{abstract}

\section{Introduction}

Luzon Strait is a rich source of eddies originating from the Kuroshio (Fig. 1). Analogous to eddies originating from the Mediterranean outflow (Meddies), the Red Sea (Reddies), and the Indonesian Throughflow (Teddies), we call those from Luzon Strait "Leddies."

Corresponding author address: Doron Nof, Oceanography, The Florida State University, 117 N. Woodward Ave., Tallahassee, FL 32306.

E-mail: nof@ocean.fsu.edu
We focus on these Leddies because, as will be seen shortly, they display unusual behavior.

\section{a. Observational background}

Farris and Wimbush (1996) were the first to point out (on the basis of SST analysis) that ring production occurs primarily during the winter. They were also the first to suggest that this fast generation may be related to the strong northeast monsoon. There is still some question as to the validity of this suggestion. For example, Jia et al. (2005) propose a "frontal instability" mechanism dependent upon Kuroshio transport (seemingly consistent 


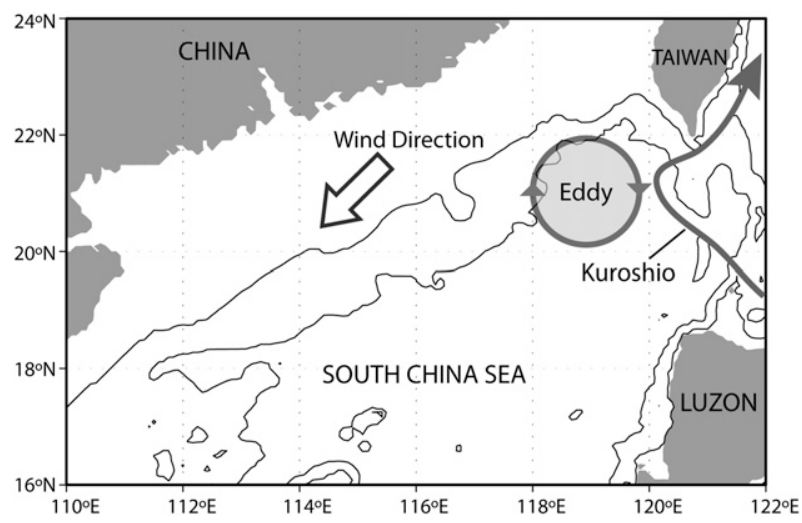

FIG. 1. Schematic diagram of a Leddy formed in Luzon Strait (adapted from Fig. 6 of Wang et al. 2008). Isobaths are for 300 and $2000 \mathrm{~m}$; the large arrow shows the direction of the northeast monsoon.

with Sheremet 2001), and the Jia and Liu (2004) satellite analysis shows 16 summer rings and 17 winter rings during 1992-2001. They concluded that “ ... the seasonal variation of eddy shedding location is unclear." Using historical hydrographic data, Qu (2000), as well as Qu et al. (2000), found that the Kuroshio intrusion reaches a maximum in winter. Using in situ hydrographic and buoy data, Wang et al. (2008) determined that, during its relatively short $\sim 40$ day lifespan, the Leddies migrate westward at a mean speed of about $10 \mathrm{~cm} \mathrm{~s}^{-1}$ but the instantaneous migration speed could reach as much as $20 \mathrm{~cm} \mathrm{~s}^{-1}$ or could be as low as $6 \mathrm{~cm} \mathrm{~s}^{-1}$.

\section{b. Theoretical background}

Although some authors (understandably) claim that Leddies migrate at the Rossby wave speed (e.g., Wang et al. 2008), we argue here that this is not really the case and suggest that, instead, the strong wind induces the observed speeds. We shall see later in section 4 that, because of the ring proximity to the equator, they are very shallow $(\sim 60 \mathrm{~m})$ and have a density anomaly of $3 \%$ (see, e.g., Qu et al. 2006). With a Coriolis parameter of $0.5 \times 10^{-4} \mathrm{~s}^{-1}$ (relevant to the Luzon Strait which is situated at $21^{\circ} \mathrm{N}$ ), the Rossby radius $R_{d}$ associated with the Leddies is about $27 \mathrm{~km}$. This needs to be distinguished from the general Rossby radius (based on a deeper general thermocline depth) that is about $40 \mathrm{~km}$ in the Luzon Strait (Cai et al. 2008).

In this context, it is appropriate to point out that, although $20^{\circ}$ is not that close to the equator, it is fairly close compared to most eddies in the World Ocean [Gulf Stream (GS), Kuroshio, and Agulhas]. (Caribbean eddies, which are an exception to the above, do not correspond to foreign fluid injected into the Caribbean, so they are not "lenses" in the sense that their thickness does not vanish along their edges. Consequently, they can be of any thickness.) The only lenslike eddies at roughly the same latitude as the South China Sea (SCS) that we know of are the Loop Current eddies in the Gulf of Mexico. These are indeed larger than Leddies because they are formed from a large fraction of the western boundary current (Yucatan Current) rather than from a small fraction of the western boundary current mass flux, as is the case in the Kuroshio.

Deciding from observations on how deep a modeled two-layer eddy should be is always very challenging because almost all eddies in the World Ocean have at least some signature all the way to the bottom of the basin. The best that one can do is guess where the maximum vertical density gradient is and use this depth as the bottom of the eddy (viz., the depth of the eddy's maximum penetration is irrelevant to the modeled depth). Using this approach, one finds that the anticyclonic eddies in the SCS are, at most, a hundred and some meters deep (see, e.g., Fig. 9b of Xiu et al. 2010), much shallower than their counterparts in the Gulf of Mexico (Maul and Vukovich 1993; Molinari and Morrison 1988) or the Gulf Stream. This is supported by the analysis of CTD (to $1000 \mathrm{~m}$ ) and ADCP data (Liu et al. 2001) as well other in situ observations ( $\mathrm{Li}$ et al. 1998).

A Rossby wave speed $\left(\beta R_{d}^{2}\right)$ of less than $1.5 \mathrm{~cm} \mathrm{~s}^{-1}$ is associated with the above Leddy Rossby radius, and this is an order of magnitude smaller than the observed 10 $20 \mathrm{~cm} \mathrm{~s}^{-1}$ mentioned above. In fact, the discrepancy between the two is even worse because the mean thickness of any anticyclone is smaller than the maximum thickness at the center, implying a migration rate smaller than the Rossby wave. [Nof (1981) argues that, for most anticyclones of that nature, it is roughly half the Rossby wave speed, giving a Leddy migration speed less than $1 \mathrm{~cm} \mathrm{~s}^{-1}$.]

The high variability of the migration rate, $6-20 \mathrm{~cm} \mathrm{~s}^{-1}$ during the relatively short 40-day lifetime (Wang et al. 2008), also places the Rossby wave idea on a shaky ground. The SCS stratification cannot possibly change so rapidly, suggesting again that it must be the wind, which has a much shorter time scale, that drives the eddies westward. Note that we will be neglecting wind effects that can produce slantwise convection (Thomas and Lee 2005) on one side and not the other, and hence may quickly (within 10-20 days) develop asymmetry.

In what follows, we will present an analytical solution for the wind-induced ring migration rate (sections 2-4) and numerical simulations (section 5) and show that both support the wind-driven Leddy idea. The numerical experiments, which are complementary to the analytical work, employ a well-tested shallow-water code that uses standard numerical techniques. The results are summarized and discussed in section 6 . 


\section{Formulation}

Consider an inviscid lens (on an $f$ plane) whose dimensions and strength in the absence of wind and $\beta$ are known. This no-wind and no- $\beta$ inviscid state is circular, steady, and stationary $(C=0)$. Both the orbital speed and thickness $h$ depend on potential vorticity and both are known. The ring has zero thickness along its rim and is floating on top of an infinitely deep resting fluid. It is then subject to a uniform surface wind stress $\tau_{s}$ and is migrating in response to this wind action. It is assumed that it does so steadily at an unknown speed $C$, forming an unknown angle $\alpha$ with the wind direction (Fig. 2). Given that the ring's time scale is $\sim o\left(f^{-1}\right)$, we expect the ring to adjust quickly to the wind.

Much can be learned at this point by vertically integrating individual terms in the equations of motion (across the lens) in a coordinate system moving steadily with the ring at speed $C$. Recall that, in a moving coordinate system, there are two Coriolis terms: one involves the migration speed $C$ and the other is associated with motions relative to the moving lens, for example,

$$
u \partial v / \partial x+\nu \partial v / \partial y+f u+f C=-g^{\prime} \partial h / \partial y+\nu \partial^{2} v / \partial z^{2},
$$

where in the conventional notation $u$ and $v$ are the horizontal speeds, $g^{\prime}$ the "reduced gravity" $g \Delta \rho_{w} / \rho_{w}$ (with $\Delta \rho_{w}$ being the density difference and $\rho_{w}$ the density), and $\nu$ is the viscosity.

First, note that a vertical integration of the Coriolis term that does not involve $C$ from the lens lower to upper boundary gives zero because the lens is an enclosed capsule (and steady) so that any Ekman flux near the surface must be compensated for by a returning flow below. This situation needs to be distinguished from that in small-amplitude (quasigeostrophic) eddies where there is a free exchange of mass between the eddy and the environmental fluid so that a surface Ekman layer is not necessarily compensated for by a return flow underneath (see, e.g., Dewar and Flierl 1987). It also needs to be distinguished from the integration of the Coriolis term involving $C$, which does not vanish but rather gives $f C h$.

Second, integration of the vertical frictional term $\left(\nu u_{z z}\right.$ or $\left.\nu v_{z z}\right)$ across the lens results in two terms: the wind-induced stress on the surface and a compensating interfacial stress on the lower interface. The latter results from the fact that the lens is migrating, whereas the fluid below and around it is stagnant. Internal horizontal friction within the lens itself does not enter the above relationship. Also, note that, since the fluid around and below the lens is infinitely deep, the wind is only affecting the lens and not the lower (and surrounding) fluid.

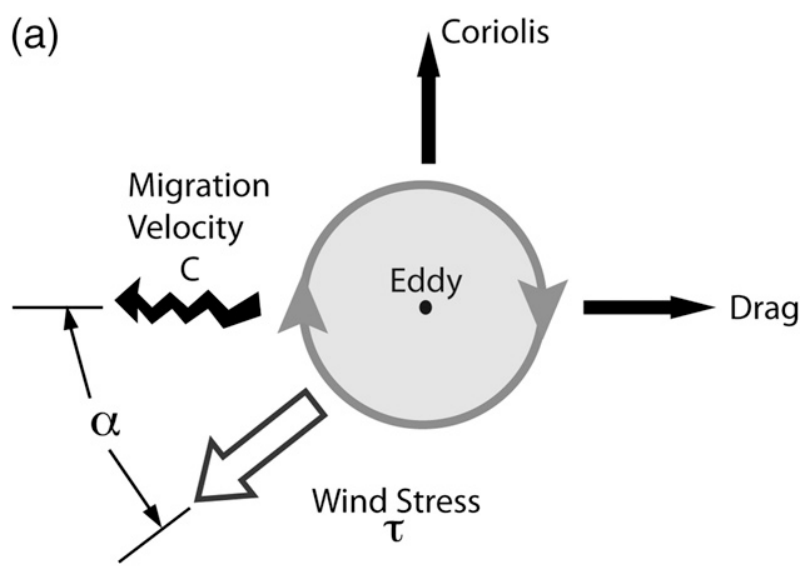

(b)
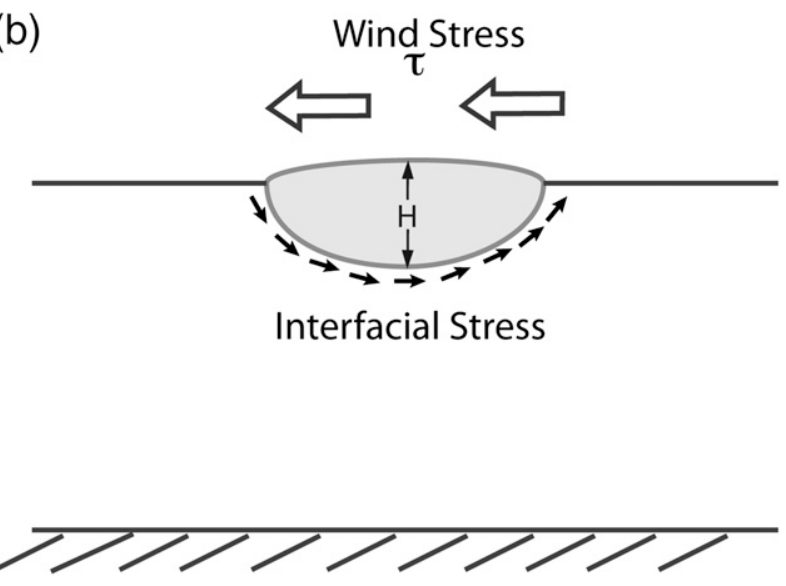

FIG. 2. Schematic diagram of (a) top view of the eddy windinduced migration and (b) a cross section of a Leddy in the direction of migration. The wind stress is the component in the drift direction $\left(\tau_{S} \cos \alpha\right)$, balanced by interfacial stress on the bottom of the lens.

In this scenario, there are three forces acting on the ring: the integrated wind stress acting on the lens upper surface pushing it in one direction, the integrated Coriolis force acting to the right of the direction of migration, and the interfacial drag acting on the lower interface in the direction opposite to the migration (Fig. 2). It is assumed that overall the wind imposes a small perturbation on the ring; that is, the resulting migration speed $C$ is small compared to the orbital speed and the distortions from a pure circle are also small.

\section{Equations}

We first note that a common expression for the frictional interfacial drag (that opposes the motion) is

$$
\iint C_{D} \mathbf{U}|\mathbf{U}| d x d y=\iint C_{D}(u+C)\left[v^{2}+(u+C)^{2}\right]^{1 / 2} d x d y
$$


where the integration is done over the area of the Leddy, $\mathbf{U}$ is the velocity vector, and $u$ and $v$ are the speeds in the $x$ and $y$ directions in the (approximately steadily) moving co- ordinate system. The coordinate system is moving with the ring at speed $C$. We expand now the term inside the integral and neglect terms $O\left(C^{2}\right)$ compared to terms of $(u C)$,

$$
\begin{aligned}
(u+C)\left(v^{2}+u^{2}+2 u C\right)^{1 / 2} & \approx(u+C)\left(u^{2}+v^{2}\right)^{1 / 2}\left[1+2 u C /\left(u^{2}+v^{2}\right)\right]^{1 / 2} \\
& \approx(u+C)\left(u^{2}+v^{2}\right)^{1 / 2}\left[1+u C /\left(u^{2}+v^{2}\right)\right] \\
& \approx u\left(u^{2}+v^{2}\right)^{1 / 2}+C\left(u^{2}+v^{2}\right)^{1 / 2}+u^{2} C /\left(u^{2}+v^{2}\right)^{1 / 2} \\
& \approx u\left(u^{2}+v^{2}\right)^{1 / 2}+C\left(2 u^{2}+v^{2}\right) /\left(u^{2}+v^{2}\right)^{1 / 2} .
\end{aligned}
$$

It is important to realize at this point that the surface integral of the first term in the last line of (2) vanishes because $u$ is asymmetrical, so only the second term is left in (1). This vanishing reflects the condition that the drag associated with the orbital speed of the lens does not enter the migration calculation on its own.

Under such conditions, there are two equations for the integrated forces acting on the ring. We derive them by integrating in the $x$ and $y$ direction over the volume. The first involves the balance in the direction of migration (wind stress component balancing interfacial drag), and the second is the balance in the direction perpendicular to the migration (wind stress component balancing Coriolis),

$$
\begin{aligned}
& \left(\tau_{S} / \rho_{W}\right) \cos \alpha \iint r d r d \theta \\
& \quad=C_{D} \iint\left(2 u^{2}+v^{2}\right)\left(u^{2}+v^{2}\right)^{-1 / 2} C r d r d \theta
\end{aligned}
$$

and

$$
\left(\tau_{S} / \rho_{W}\right) \sin \alpha \iint r d r d \theta=f C \iint h r d r d \theta
$$

where $h$ is the ring thickness, $C_{D}$ the interfacial drag coefficient, and the surface integration (in polar coordinates) is done from zero to $R$ (the ring radius) and zero to $2 \pi$. These two equations give the solution for the two unknowns, $C$ and $\alpha$. The reader who wondered about the pressure term is reminded that there is no net pressure force acting on the lens because the thickness vanishes around the rim. Namely, using Green's theorem, the integration of the pressure term over the lens yields

$$
\iint \frac{\partial}{\partial y}\left(h^{2} / 2\right) d x d y=-\frac{1}{2} \oint h^{2} d x=0
$$

where the integration along the boundary (rhs) is done in a counterclockwise manner (though this does not really matter because $h=0$ along the boundary). Similarly, the integration of the nonlinear terms also vanishes. The contribution of the variation of the Coriolis parameter with latitude, $\beta$, is negligibly small because, as already pointed out earlier (using scaling), the Rossby wave speed introduced by $\beta$ is much smaller than our wind-induced speed. This is verified later using our detailed solution.

\section{Solution}

Dividing (4) by (3) gives

$\tan \alpha=f \iint h r d r d \theta / C_{D} \iint\left(2 u^{2}+v^{2}\right)\left(u^{2}+v^{2}\right)^{-1 / 2} r d r d \theta$,

which can be used to calculate the angle because the eddy structure is known in advance (from the no-wind case). We observe that the direction of migration is independent of the wind stress $\tau_{s}$.

Taking the simple case for which the orbital speed $v_{\theta}$ is linear,

$$
v_{\theta}=-\gamma f r / 2, \quad \gamma \leq 1
$$

where $\gamma$ is the vorticity divided by $f$, and using

$$
\frac{v_{\theta}^{2}}{r}+f v_{\theta}=g^{\prime} \frac{d h}{d r}
$$

we find that the ring volume and radius are

$$
\begin{aligned}
V & =\gamma(2-\gamma) \pi f^{2} R^{4} / 16 g^{\prime} ; \quad R=2 \sqrt{2} R_{d} / \gamma^{1 / 2}(2-\gamma)^{1 / 2} \\
R_{d} & =\sqrt{g^{\prime} H} / f
\end{aligned}
$$

where $H$ is the ring's maximum thickness at the center. For $\gamma=1$, the system corresponds to a zero potential vorticity eddy and, in the more general case, the potential vorticity is not uniform. In the zero potential vorticity case, 


$$
V=4 \pi f^{2} R_{d}^{4} / g^{\prime}=64 \pi\left(g^{\prime}\right)^{2} H^{2} / f^{4} ; \quad h=H-f^{2} r^{2} / 8 g^{\prime},
$$

showing immediately that, for a fixed volume, reducing $f$ (i.e., getting closer to the equator) implies smaller $H$ (proportional to $f^{2}$ ).

The denominator in (5) gives $\pi \gamma C_{D} f R^{3} / 2$, whereas the numerator in (5) is just $f$ times the lens volume, so (5) ultimately gives our desired expression for $\alpha$,

$$
\tan \alpha=(2-\gamma) f^{2} R / 8 g^{\prime} C_{D}
$$

Several limits should now be considered. First, small rings $($ small $R$ ) will move in the direction of the wind, whereas large rings will move at $90^{\circ}$ to the right. Most rings will be in between those two limits. A highly frictional interface (large $C_{D}$ ) also corresponds to rings moving in the direction of the wind, and a weakly frictional interface corresponds to rings moving at $90^{\circ}$ to the right. As the equator is approached $(f \rightarrow 0)$, the ring migration approaches the direction of the wind.

For a $120-\mathrm{km}$ radius ring, a Coriolis parameter of $0.5 \times$ $10^{-4} \mathrm{~s}^{-1}$ (relevant to Luzon Strait), $\gamma=0.2$ (corresponding to a vorticity of $0.1 f$ ), and $C_{D}=0.002$, the tilt relative to the wind direction will be roughly $48^{\circ}$ to the right. Here, the Rossby radius is about $27 \mathrm{~km}$ and, due to the low latitude, the central depth $H$ is only $60 \mathrm{~m}$. Once $\alpha$ is known, the speed can be calculated from either (3) or (4). Noting that the ratio between the two integrals in (4) is $H / 2$ regardless of the vorticity $\gamma$ and using $\tau_{S}=\rho_{A} C_{D A} U_{10}^{2}$, where the subscript $A$ corresponds to air (so that $C_{D A}$ is the air/water drag coefficient), we find from (4),

$$
C=2\left(\rho_{A} / \rho_{W}\right) C_{D A} U_{10}^{2}(\sin \alpha) / f H,
$$

which is valid for all $\gamma$. Since $\alpha$ depends on $\gamma,(10)$ is not truly independent of $\gamma$. Also note that, for small alpha, $f$ does not explicitly appear in the expression for the migration rate but it enters the expression implicitly through $H$, which is small at low latitudes. For the parameters mentioned above, $U_{10}$ of $15 \mathrm{~m} \mathrm{~s}^{-1}$ (strong monsoon wind) and a wind/water drag coefficient of, again, 0.002, $C$ is $\sim 22 \mathrm{~cm} \mathrm{~s}^{-1}$, which is very reasonable. Here the maximum orbital speed is $60 \mathrm{~cm} \mathrm{~s}^{-1}$, which, as required, is larger than the migration rate.

To summarize this subsection, it can be seen from (10) that the effects of winds on lenses can only be important when the rings are shallow (small $H$ ), the latitude is small (small $f$ ), and the winds are strong (high $U_{10}$ ). With high interfacial drag the rings move slowly in the wind direction, whereas with low drag they move fast $90^{\circ}$ to the right. This situation seems to hold in Luzon Strait but will not hold for Gulf Stream rings where the effect will most likely be three orders of magnitude smaller because the lenses involve a very large amount of fluid, so $H$ is much larger. Also, $f$ is larger and $U_{10}$ is much smaller. We shall return to this important point later.

\section{Numerical simulations}

The numerical model is the shallow-water, isopycnic coordinate general circulation model of Bleck and Boudra (1986). The advantage of this coordinate system is that lateral diffusion is along isopycnal surfaces where mixing of material properties by eddies in the stably stratified parts of the oceans mostly occurs (Chassignet et al 1990). The suitability of this model for ocean eddies studies was examined by Chassignet et al. (1990) and Chassignet and Cushman-Roisin (1991).

The model consists of a momentum equation and a continuity equation,

$$
\begin{aligned}
& \frac{\partial \mathbf{V}}{\partial t}+\frac{\nabla_{\rho} \mathbf{V}^{2}}{2}+(\varsigma+f) \mathbf{k} \times \mathbf{V} \\
& =-\nabla_{\rho} M+\delta \frac{\partial \tau}{\partial z}+A_{M} h^{-1} \nabla_{\rho} \cdot\left(h \nabla_{\rho} \mathbf{V}\right)
\end{aligned}
$$

and

$$
\frac{\partial h}{\partial t}+\nabla_{\rho} \cdot(h \mathbf{V})=0
$$

where $M=g z+p \delta$ is the Montgomery potential, $h$ is the thickness of a layer of constant density, $\delta$ is the specific volume $\rho^{-1}, \tau$ is the stress, $A_{M}$ is lateral viscosity, and the subscript $\rho$ indicates derivatives on surfaces of constant density. For more details, the reader is referred to Bleck and Boudra (1986).

The model is configured in a two-layer $2000 \mathrm{~km} \times$ $2000 \mathrm{~km}$ square domain on an $f$ plane with a grid spacing of $10 \mathrm{~km}$. It is initialized with a Gaussian distribution of the upper-layer thickness, $h=H e^{-r^{2} / 2 L^{2}}$, where $r$ is the radius (measured from the center of the lens), $L$ is the radius of maximum velocity, and $H$ is the interface thickness at the lens center. The upper-layer thickness $h$ is zero at the edge of the lens. Here, we do not use the analytical expression for the thickness for two reasons. First, introducing the analytical speed and thickness to the numerics will cause local instabilities around the rings edge because the numerics cannot easily handle discontinuities in either the magnitude of the speed and thickness or their gradients. Second, since the analytical expression for the (critical) Coriolis force involves surface integrals of $h$ over the entire eddy, there is hardly any 


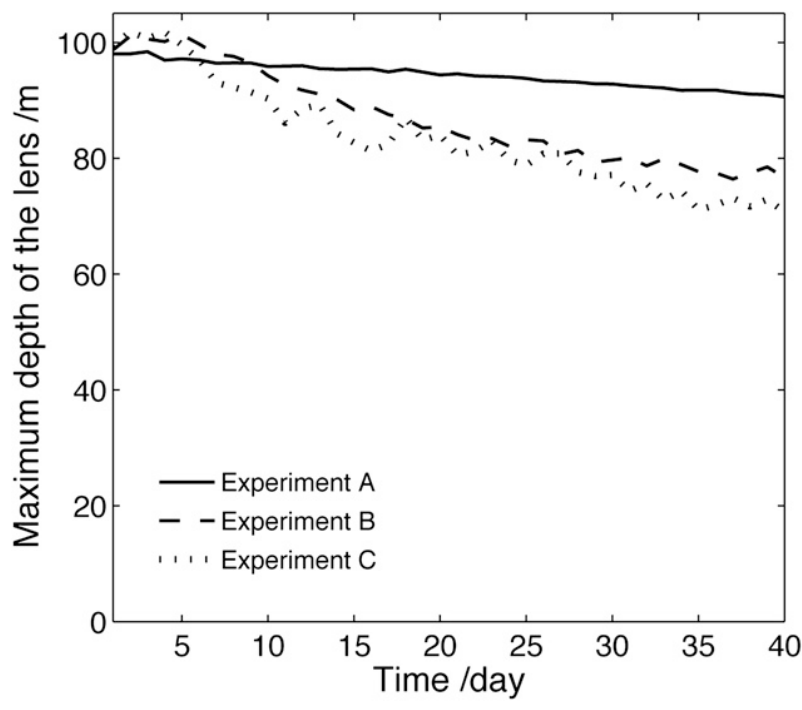

FIG. 3. Maximum eddy thickness ( $m$ ) as a function of time for the three numerical experiments: A (solid line; $C_{D}=0$ ), B (dashed line; $\left.C_{D}=0.001\right)$, and C (dotted line; $\left.C_{D}=0.002\right)$.

contribution at all from the region in the immediate vicinity of the rim where the thickness goes to zero. Consequently, at least for the Coriolis force, it does not really matter whether the edge is represented accurately.

The ring radius and $H$ are taken as $140 \mathrm{~km}$ and $100 \mathrm{~m}$, resembling the anticyclonic eddies separated from the Kuroshio in Luzon Strait. The reduced gravity $g^{\prime}$ is taken to be (on the high side) $0.0294 \mathrm{~m} \mathrm{~s}^{-2}$, corresponding to density variation of 3\% [see, e.g., Qu et al. (2006), their Fig. 7a, showing water density changes from 22 to 27 in the upper $1000 \mathrm{~m}]$. The initial second (lower) layer thickness is $5000 \mathrm{~m}$, which is 50 times larger than the maximum thickness of the lens. The model is forced with zonal wind of $0.5 \mathrm{~N} \mathrm{~m}^{-2}$, which is the typical value of a winter monsoon in the SCS, corresponding to a wind speed of $15 \mathrm{~m} \mathrm{~s}^{-1}$ at 10-m height (see, e.g., Liu and Xie 1999). The Coriolis parameter was taken to be $0.5 \times$ $10^{-4} \mathrm{~s}^{-1}$. In addition to the small drag that the model contains for stability purposes, a quadratic drag is applied to the interface between the two layers. To avoid a significant reduction of the orbital speed by this additional drag, it was applied only to the migration speed of the lens but not the orbital speed [i.e., in accordance with the analysis presented in the beginning of section 3 , the first term in the last line of (2) was ignored].

Three numerical experiments, differing in the value of the drag coefficient $C_{D}$, were executed. As each individual experiment includes numerous data points, we feel that this number of experiments is adequate. The quadratic drag coefficient $C_{D}$ is zero in experiment $\mathrm{A}$, 0.001 in experiment $\mathrm{B}$, and 0.002 in experiment $\mathrm{C}$. The

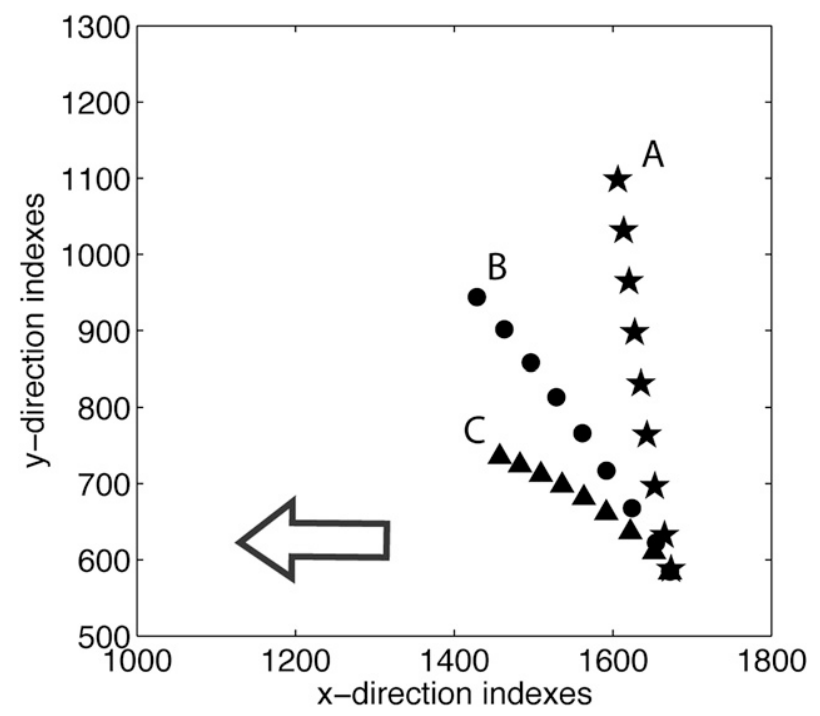

FIG. 4. Ring numerical trajectories calculated from the center of mass (every 5 days from day 1 to day 60). Stars, circles, and triangles mark the trajectories of experiments $\mathrm{A}, \mathrm{B}$, and $\mathrm{C}$. The large arrow marks the direction of the wind. Note that high-drag rings (C) are more aligned with the wind direction than the low-drag rings (A) but, as expected, migrate more slowly.

numerical results are shown in Figs. 3-8. Figure 3 displays the decay of the central thickness, which comes about through flattening of the eddy. This flattening is due to both numerical friction, which, as mentioned, is added for stability, and physical interfacial drag $C_{D} \mathbf{U}|\mathbf{U}|$, which is generated in response to the strong wind. It is similar to the analytics and presumably the ocean.

Figure 3 shows that, under these conditions, the central thickness decreases with time owing to the two frictional terms, but the reduction is not very significant $(<30 \%)$. Figure 4 illustrates the migration path. It vividly shows that, as the analytics predicts, increasing the friction (interfacial drag) slows down the rings and decreases their deflection to the right. Conversely, decreasing the interfacial friction speeds the migration and increases the deflection. Figures 5-7 display a comparison of the numerical and analytical migration angle (right panels) and the migration speeds (left panels). The agreement of the angles is excellent (deviations are less than $\sim 5 \%$ ), and the agreement of the speeds is good (deviations are less than $\sim 30 \%$ ).

As should be the case, the numerical speed is always smaller than the analytical (because of the numerical friction). Figure 8 shows the thickness contours as a function of time for our largest friction experiment. In a sense, this is our "worst" experiment. It illustrates that the ring breaks down and splits into two rings (one large and one small) on day 20. The issue of numerical eddy splitting is a difficult one. From a theoretical point of 

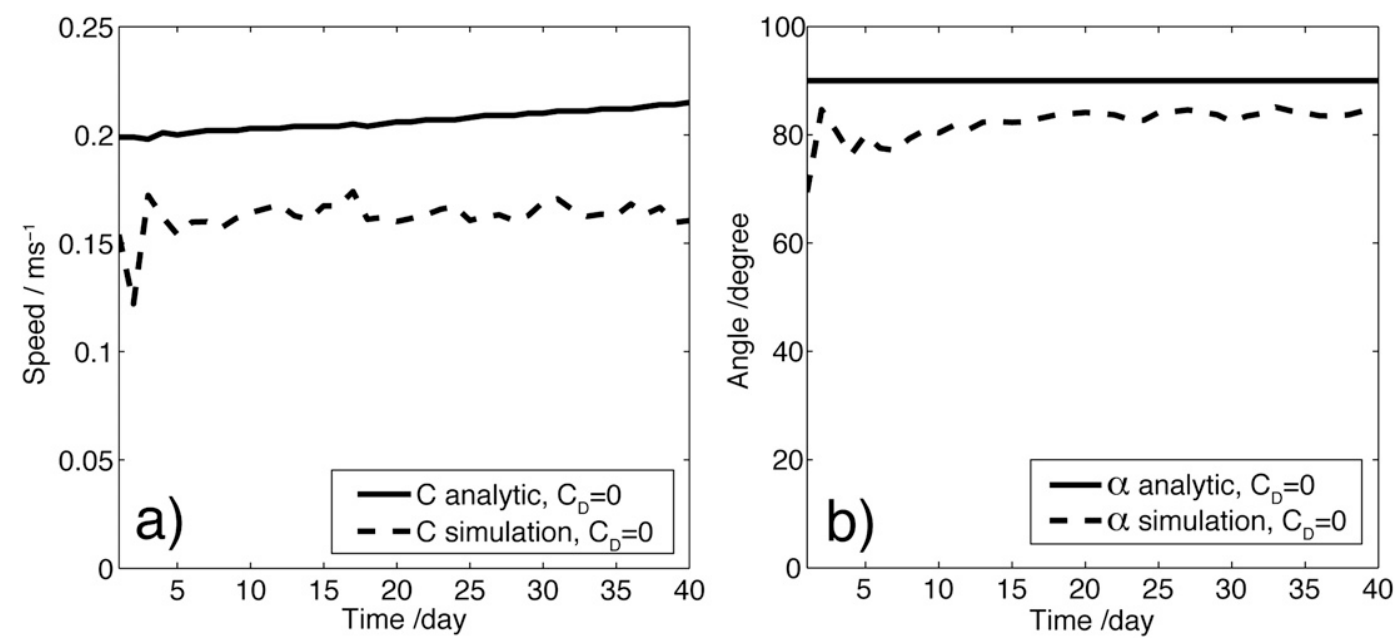

FIG. 5. (left) Analytical (solid line) and numerical migration speed (dashed line) of expt A. (right) Angle of migration $\alpha$ of expt A.

view, inviscid anticyclones in a reduced gravity model cannot possibly split (Nof 1990, 1991), but numerical eddies do occasionally split (Drijfhout 2003), perhaps due to friction. To our knowledge, there is no clear explanation as to why and when it happens, and there are not that many observations of actual eddies splitting to help us resolve this issue. We included the splitting in our plots merely because this is what the numerical model does.

\section{Summary and discussion}

In contrast to most rings and eddies in the World Ocean, which do not display a strong seasonal variability, the Luzon Strait eddies (Fig. 1) exhibit a pronounced variability with many more eddies being formed during the winter than during the summer. Because the stratification is usually smaller in the winter, it is unlikely that this enhanced rings generation is due to increased instability. Instead, as suggested by the observational analysis of Farris and Wimbush (1996) and later by the regional numerical work of Metzger and Hurlburt (1996) and Zhao et al. (2009), it could be due to the strong northeast monsoon, which blows toward the southwest during winter, forcing the Kuroshio water westward into the SCS. Admittedly, these arguments are not entirely convincing (regarding the generation mechanism) because none of the above papers have addressed the generation process directly. For example, Metzger and Hurlburt (1996) is a coarse-resolution modeling effort, whereas Farris and Wimbush (1996) do not offer a dynamical explanation.
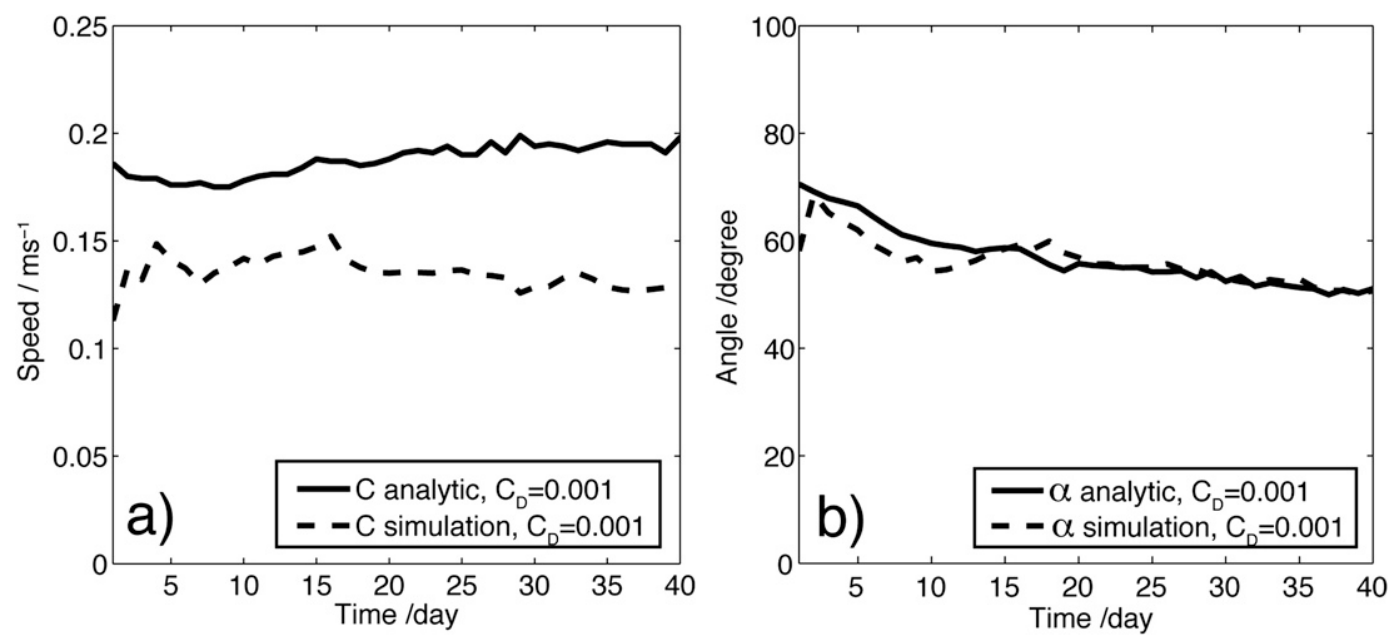

FIG. 6. As in Fig. 5 but for expt B. 

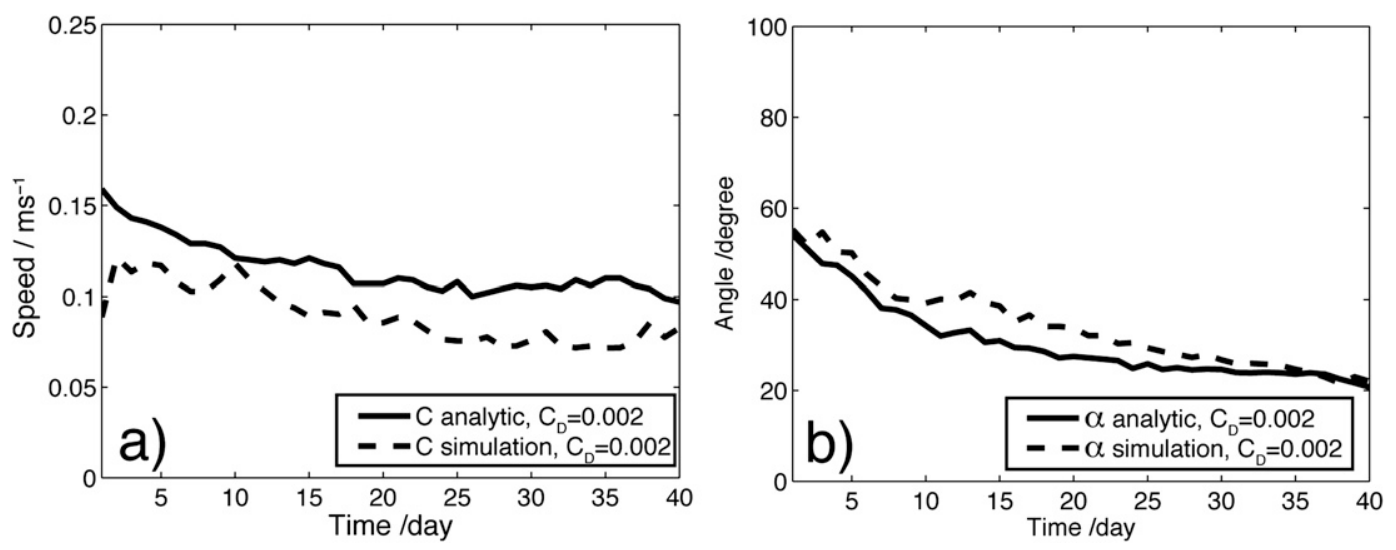

FIG. 7. As in Fig. 5 but for expt C.

A recent paper (Jia and Chassignet 2011) shows that the use of an inaccurate mean sea surface height in Jia and Liu (2004) led to the misidentification of eddy shedding events during summer. Jia and Chassignet now use altimeter data and output from the Hybrid Coordinate Ocean Model global assimilation run to show that that most eddy shedding events occur from December through March. Furthermore, while Sheu et al. (2010) did not directly address the eddy shedding process, they agree that the Kuroshio intrusion is stronger in winter than in summer.

The fact that we provide a plausible explanation for why the migration speed of Leddies is much greater than the local Rossby wave speed supports our idea that the migration is due to the wind. As pointed out earlier in the text, the typical migration speed of a detached eddy in the SCS is about $10 \mathrm{~cm} \mathrm{~s}^{-1}$ in winter [Wang et al. (2008); see their Fig. 12b, where eddy AE2 was derived from the sea level anomaly maps provided by $\mathrm{Hu}$ et al. (2001), using altimetry]. This speed is much greater than the local first mode baroclinic Rossby wave speed ( $5 \mathrm{~cm} \mathrm{~s}^{-1}$, according to Cai et al. 2008, their Fig. 3).

A rough calculation indicates that the northeast monsoon winds are so strong that (through their Ekman flux) they can produce up to two Leddies in three months. Here, we use an analytical approach as well as processoriented numerical models to show that these winds induce a very fast eddy migration rate, enabling the newly formed Leddies to quickly escape from the generation area (near the strait), thus freeing the region for the formation of the next ring in line. This process enhances the production of rings because it allows them to form much faster.

Although some authors suspected that Leddies migrate at the Rossby wave speed, we argue here that this is not really the case and suggest instead that the strong wind induces the observed speeds. We note first that a Rossby wave speed $\left(\beta R_{d}^{2}\right)$ less than $1.5 \mathrm{~cm} \mathrm{~s}^{-1}$ is associated with the Leddy Rossby radius, and this is an order of magnitude smaller than the observed 10 $20 \mathrm{~cm} \mathrm{~s}^{-1}$ mentioned above. Second, we note that the high variability of the migration rate, $6-20 \mathrm{~cm} \mathrm{~s}^{-1}$ during the relatively short 40-day lifetime (Wang et al. 2008), also places the Rossby wave idea on a questionable ground. The SCS stratification cannot possibly change so rapidly, suggesting again that it must be the wind, which has a much shorter time scale, that drives the eddies westward.

We chose to look at a lens, rather than the familiar small-amplitude quasigeostrophic eddy, because Leddies are associated with the injection of foreign warm and fresh water into the SCS. From a topological point of view, such injection implies that only eddies that completely encapsulate the anomalous water can exist in the SCS. Namely, we consider eddies containing warm water whose thickness is finite at the center but zero along the rim. These eddies are embedded in a very deep layer of slightly heavier water (Fig. 2) and are inherently nonlinear because $\left(v_{\theta} / f r\right) \sim o(1)$ and the amplitude variation is $O(1)$.

Using the balance of forces in the direction of migration as well as the direction perpendicular to it and assuming that the wind stress acting on the Leddies is compensated for by interfacial stress along the ring lower boundary, we derived simple relationship for the migration speed as well as the migration angle relative to the wind direction (relations 9 and 10). They show that, with high interfacial friction, the Leddies move relatively slowly (but still fast relative to a Rossby wave) in the direction of the wind, whereas with low interfacial friction they move very fast at $90^{\circ}$ to the right of the wind (looking downwind). They also show that small rings move faster than larger rings because they are lighter and, hence, are more susceptible to the wind. 

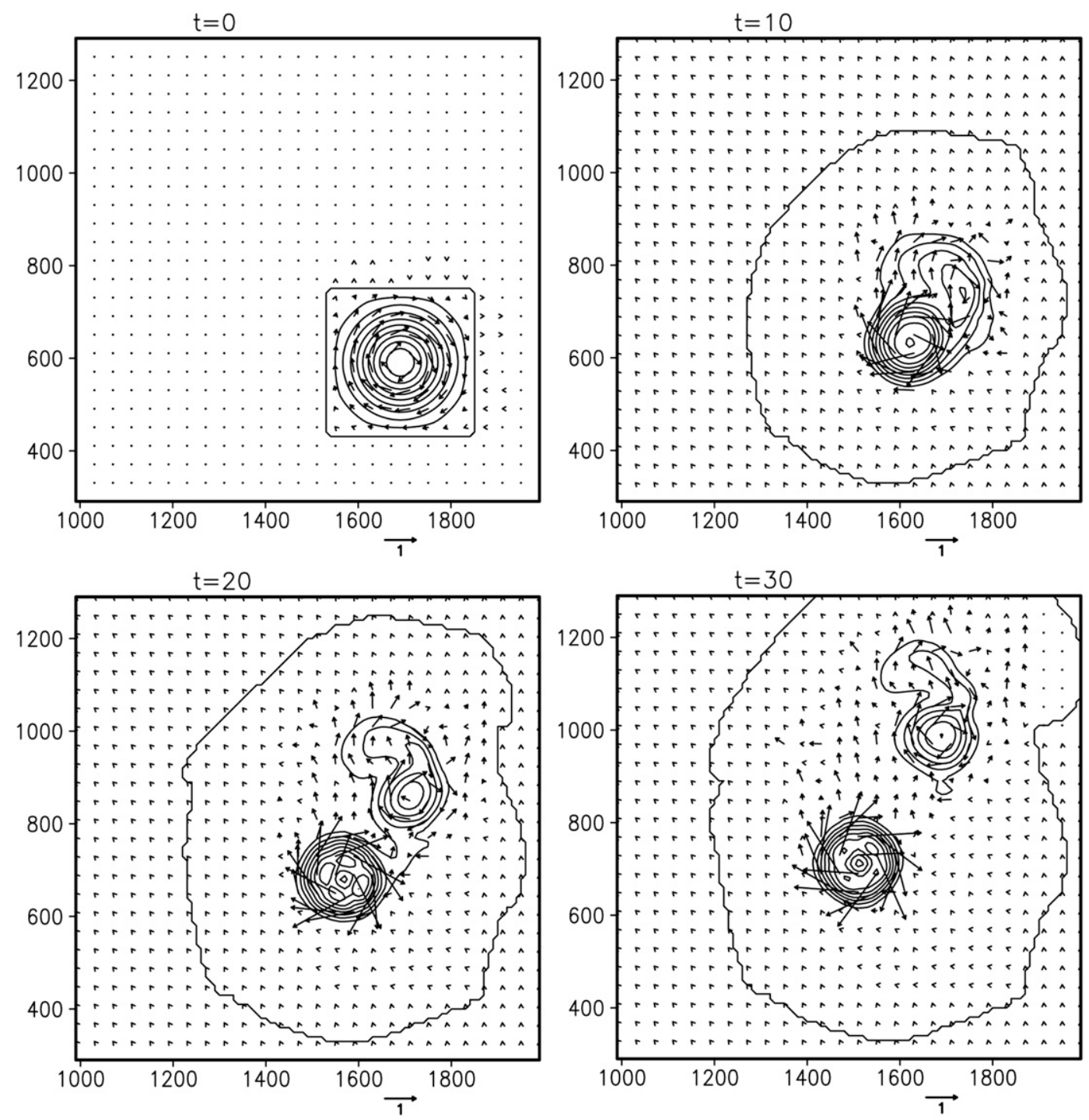

FIG. 8. Thicknesses (m, interval $10 \mathrm{~m}$ ) and velocity $\left(\mathrm{m} \mathrm{s}^{-1}\right.$ ) of expt $\mathrm{C}$ (from day zero to day 30 in 10-day increments), contour interval $10 \mathrm{~m}$. Note that this large friction experiment is the worst of our three experiments.

One can easily see from our solution that most rings in the World Ocean (e.g., Gulf Steam rings, Agulhas rings) will not be subject to a very significant wind effect because the wind is in general relatively light and the rings are usually fairly large. Specifically, with $H \sim$ $500-1000 \mathrm{~m}$ and wind at $10 \mathrm{~m}$ of $3-5 \mathrm{~m} \mathrm{~s}^{-1}$, the rings will migrate at a speed on the order of $1 \mathrm{~mm} \mathrm{~s}^{-1}$. In that sense, Leddies are an anomaly because they are shallow $(<100 \mathrm{~m})$, they are in low latitude $\left(\sim 20^{\circ} \mathrm{N}\right)$, and they are subject to strong monsoon winds $\left(U_{10} \sim 10\right.$ $20 \mathrm{~m} \mathrm{~s}^{-1}$ ). As a result, their wind-induced migration is much larger, roughly $10-20 \mathrm{~cm} \mathrm{~s}^{-1}$. For a $120-\mathrm{km}$ radius ring, a Coriolis parameter of $0.5 \times 10^{-4} \mathrm{~s}^{-1}$, $\gamma=0.2$ (corresponding to a vorticity of $0.1 f$ ), and $C_{D}=0.002$, the tilt relative to the wind direction will be roughly $48^{\circ}$ to the right. Given that the northeast monsoon is blowing toward the southwest, this $48^{\circ}$ tilt implies that the Leddies migrate to the west, just like Rossby waves do but for a different reason. Because of the topography and the orientation of the boundary in the SCS (Fig. 1), the Leddies cannot maintain this westward migration for very long. Fairly quickly, they adjust to the topography and start migrating toward the southwest.

We also noted that Leddies are formed from a fraction of the Kuroshio that manages to penetrate into the SCS, whereas Gulf Stream (GS) rings are from the entire GS, which meanders and closes upon itself. Consequently, Leddies have much less volume than Gulf Steam rings and, because they are closer to the equator, are also 
much shallower. The winds over the GS are indeed strong (as high as $\sim 8 \mathrm{~m} \mathrm{~s}^{-1}$ ), but they are not as strong as the monsoon $\left(\sim 15 \mathrm{~m} \mathrm{~s}^{-1}\right)$, implying that the wind stress during a monsoon is still four times greater in the SCS than it is over the GS. Also, the Leddy thickness is $1 / 10$ of the GS rings depth and $f$ is half, giving a Leddy migration speed that is 80 times that of the GS rings [because the migration rate is roughly $\left.2\left(\tau_{S} / \rho_{W}\right)(\sin \alpha) / f H\right]$.

We then proceeded with a series of process-oriented numerical experiments in which there were two kinds of frictional terms: the familiar, very small (and hopefully negligible) numerical friction, which is included merely for numerical stability, and a physical interfacial friction, $C_{D} \mathbf{U}|\mathbf{U}|$, which is similar to our analytics and (hopefully) nature because the migration speeds are very high. As mentioned, to avoid unnecessary spindown in the numerics, we eliminated the first term in the last line of (2) from the numerical model. This term vanishes in the analytics due to symmetry and does not enter the balances associated with the migration.

Our numerical results are shown in Figs. 3-8. All show excellent agreement between the predicted analytical and numerical direction of migration (differences of less than $5 \%$ ) and reasonable agreement of the migration speeds (discrepancies of $<30 \%$ ). As should be the case, the numerical speeds are all smaller than the analytical owing to numerical friction, which slows the rings down. In general, the small interfacial friction experiments (i.e., small $C_{D}$ ) are in better agreement with the analytics, probably because the rings stay more coherent during the runs. The numerical viscosity is, of course, not totally negligible, but it is not that bad because it is solely used for numerical stability. Our worst agreement, which is shown in Fig. 8, involves splitting of the ring into a large ring and a small ring at day 20. Despite that split, the agreement is still reasonable.

Finally, it should be recalled that, while the analytical/ numerical comparison is useful, it does not give the final answer to the Leddy drift question. Both the numerical experiments and analytical models have their drawbacks and strengths. On one hand, the numerical models incorporate a wider range of processes but, on the other hand, simple analytical models are more amenable to analysis and are valuable for their illumination of any changes that take place in response to other variations. The ultimate answer to the question of how rings move owing to strong wind action can only be provided by a careful analysis of both satellite and in situ observations. Given the mature state of satellite altimetry, scatterometry, and other satellite-based techniques, it is hoped that both methods will be available soon.
Acknowledgments. Y. Jia is supported by National Key Basic Research Project of China (2007CB411803), Natural Science Foundation of China (40706005). Dr. Nof and Dr. Chassignet's research is supported by NSF Grants OCE-0752225 and ARC-0902835. The Binational Science Foundation (2006296) is also supporting Dr. Nof's work.

\section{APPENDIX}

\section{Symbols}

\begin{tabular}{ll}
$A_{M}$ & Lateral viscosity \\
$C$ & Migration speed of the eddy's center \\
$C_{D A}$ & Coefficient of interfacial drag in the \\
& atmosphere \\
$C_{D}$ & Coefficient of interfacial drag \\
$f$ & Coriolis parameter \\
$g^{\prime}$ & Reduced gravity \\
$\mathrm{GS}$ & Gulf Stream \\
$H$ & Eddy maximum thickness \\
$h$ & Thickness of the eddy \\
$L$ & Radius of maximum velocity in the \\
& eddy \\
$r, \theta$ & Polar coordinates \\
$R$ & Radius of the eddy \\
$R_{d}$ & Rossby radius \\
$\mathrm{SCS}$ & South China Sea \\
$u, v$ & Horizontal velocity components \\
$U_{10}$ & Wind speed at $10 \mathrm{~m}$ above ocean \\
\multicolumn{3}{c}{ surface } \\
$v_{\theta}$ & Orbital speed of the eddy \\
$\alpha$ & Migration angle of the eddy center \\
$\rho_{A}$ & Density of the atmosphere \\
$\rho_{w}, \Delta \rho_{w}$ & Density of the ocean and density \\
$\tau_{S}$ & difference between the layers \\
$\delta$ & Surface wind stress \\
$\gamma$ & Specific volume \\
$\nu$ & Vorticity of the eddy divided by $f$ \\
& Viscosity \\
\end{tabular}

\section{REFERENCES}

Bleck, R., and D. Boudra, 1986: Wind-driven spin-up in eddyresolving ocean models formulated in isopycnic and isobaric coordinates. J. Geophys. Res., 91, 7611-7621.

Cai, S., X. Long, R. Wu, and S. Wang, 2008: Geographical and monthly variability of the first baroclinic Rossby radius of deformation in the South China Sea. J. Mar. Syst., 74 (1-2), 711-720.

Chassignet, E. P., and B. Cushman-Roisin, 1991: On the influence of a lower layer on the propagation of nonlinear oceanic eddies. J. Phys. Oceanogr., 21, 939-957.

- D. B. Olson, and D. B. Boudra, 1990: Motion and evolution of oceanic rings in a numerical model and in observations. J. Geophys. Res., 95, 22 121-22 140.

Dewar, W. K., and G. Flierl, 1987: Some effects of the wind on rings. J. Phys. Oceanogr., 17, 1653-1667. 
Drijfhout, S. S., 2003: Why anticyclones can split. J. Phys. Oceanogr., 33, 1579-1591.

Farris, A., and M. Wimbush, 1996: Wind-induced Kuroshio intrusion into the South China Sea. J. Oceanogr., 52, 771-784.

Hu, J., H. Kawamura, H. Hong, F. Kobashi, and D. Wang, 2001: 3-6 months variation of sea surface height in the South China Sea and its adjacent ocean. J. Oceanogr., 57, 69-78.

Jia, Y., and Q. Liu, 2004: Eddy shedding from the Kuroshio bend at Luzon Strait. J. Oceanogr., 60, 1063-1069.

— shedding from the Kuroshio intrusion in the Luzon Strait. $J$. Oceanogr., in press.

—, Q. Liu, and W. Liu, 2005: Primary study of the mechanism of eddy shedding from the Kuroshio bend in Luzon Strait. J. Oceanogr., 61, 1017-1027.

Li, L., W. D. Nowlin Jr., and J. L. Su, 1998: Anticyclonic rings from the Kuroshio in the South China Sea. Deep-Sea Res. I, 45, 1469-1482.

Liu, Q., Y. Jia, P. Liu, Q. Wang, and P. C. Chu, 2001: Seasonal and intraseasonal thermocline variability in the central South China Sea. Geophys. Res. Lett., 28, 4467-4470.

Liu, W. T., and X. Xie, 1999: Spacebased observations of the seasonal changes of south Asian monsoons and oceanic responses. Geophys. Res. Lett., 26, 1473-1476.

Maul, G. A., and F. M. Vukovich, 1993: The relationship between variations in the Gulf of Mexico Loop Current and Straits of Florida volume transport. J. Phys. Oceanogr., 23, 785-796.

Metzger, E. J., and H. E. Hurlburt, 1996: Coupled dynamics of the South China Sea, the Sulu Sea and the Pacific Ocean. J. Geophys. Res., 101, 12 331-12 352.

Molinari, R. L., and J. Morrison, 1988: The separation of the Yucatan Current from the Campeche Bank and the intrusion of the Loop Current into the Gulf of Mexico. J. Geophys. Res., 93, $10645-10654$.
Nof, D., 1981: On the beta-induced migration of isolated baroclinic eddies. J. Phys. Oceanogr., 11, 1662-1672.

- 1990: The role of angular momentum in the splitting of isolated eddies. Tellus, 42, 469-481.

_ 1991: Fission of single and multiple eddies. J. Phys. Oceanogr., 21, 40-52.

Qu, T., 2000: Upper-layer circulation in the South China Sea. J. Phys. Oceanogr., 30, 1450-1460.

- H. Mitsudera, and T. Yamagata, 2000: Intrusion of the North Pacific waters into the South China Sea. J. Geophys. Res., 105, 6415-6424.

— J. B. Girton, and J. A. Whitehead, 2006: Deepwater overflow through Luzon Strait. J. Geophys. Res., 111, C01002, doi:10.1029/ 2005JC003139.

Sheremet, V. A., 2001: Hysteresis of a western boundary current leaping across a gap. J. Phys. Oceanogr., 31, 1247-1259.

Sheu, W., C. Wu, and L. Oey, 2010: Blocking and westward passage of eddies in the Luzon Strait. Deep-Sea Res. II, 57, 1783-1791, doi:10.1016/j.dsr2.2010.04.004.

Thomas, L. N., and C. M. Lee, 2005: Intensification of ocean fronts by down-front winds. J. Phys. Oceanogr., 35, 10861102.

Wang, Q., S. Danilov, and J. Schröter, 2008: Finite element ocean circulation model based on triangular prismatic elements, with application in studying the effect of topography representation. J. Geophys. Res., 113, C05015, doi:10.1029/ 2007JC004482.

Xiu, P., F. Chai, L. Shi, H. Xue, and Y. Chao, 2010: A census of eddy activities in the South China Sea during 1993-2007. J. Geophys. Res., 115, C03012, doi:10.1029/2009JC005657.

Zhao, W., Y. Hou, P. Qi, L. E. Ken-tang, and M. K. Li, 2009: The effects of monsoons and connectivity of South China Sea on the seasonal variations of water exchange in the Luzon Strait. J. Hydrodyn., 21, 264-270. 